\title{
GESNERUS
}

Vierteljahrsschrift für Geschichte der Medizin und der Naturwissenschaften Revue trimestrielle d'histoire de la médecine

Jahrgang/Vol. $25 \quad 1968$ Heft/Fasc. 1/2

\section{Zum 25. Jahrgang des Gesnerus}

Im 25. Jahr des Erscheinens der Vierteljahrsschrift Gesnerus ihres Anfanges zu gedenken und kurze Rückschau zu halten, hat der Herausgeber der Zeitschrift Veranlassung genug. Gilt es doch, Rechenschaft darüber abzulegen, ob die Ziele, die bei der Gründung der Vierteljahrsschrift ins Auge gefaßt worden sind, im Laufe der 25 Jahre eingehalten werden konnten. Das erste Heft des ersten Jahrganges, das 1943 herauskam, gibt uns gleichzeitig die Antwort darauf: das Heft enthält zwei Nekrologe: den Nachruf auf Jean Strohl, den Begründer der Zeitschrift in Zollikon (Zürich), und denjenigen auf Arnold C. Klebs in Nyon, den weltbekannten Inkunabelnforscher.

Ich darf sagen: Es war für den Herausgeber einer neuen Zeitschrift, welche von dem aus einzigartiger Fülle schöpfenden Biologiehistoriker JEAN Sтroнl geleitet, in lebendig-freundschaftlichem Zusammenwirken hätte wachsen und reifen sollen, schwer, das Unternehmen ohne den fördernden Impuls des Gefährten und Meisters zu beginnen, welcher in generöser Freigebigkeit sein reiches Wissen stets mit andern zu teilen bereit war.

Arnold C. Klebs in Nyon, welcher bald leise mahnend, bald vernehmlich pochend auf die Notwendigkeit der Gründung einer schweizerischen medizin- und naturwissenschaftshistorischen Zeitschrift hingewiesen und ihrem Werden helfend zur Seite gestanden hat, wurde durch den Tod abberufen, bevor die kleine Zeitschrift das Licht der Welt erblickte.

Jean Strohl (1886-1942) hat die Herausgabe des ersten Gesnerus-Heftes nicht erlebt. Von ihm stammt die wegweisende Einführung des ersten Heftes - er schrieb sie im Sommer 1942 - um bald darauf, am 7.Oktober 1942, im Alter von noch nicht 57 Jahren, vom Tode dahingerafft zu werden.

Es liegt mir daran, einige Abschnitte aus dieser Einführung hier wiederzugeben, weil daraus hervorgeht, mit welch weit gespannten Gedanken Jean Strohl die Herausgabe einer solch kleinen Zeitschrift begleitete. Seinem In- 
genium wäre es auch gelungen, der Zeitschrift einen geistigen Gehalt zu sichern, wie es einer Neugründung angemessen gewesen wäre.

In der Einführung Jean Strohls heißt es: «Wenn unter besonders schwierigen, die ganze Welt bedrückenden Verhältnissen es hier unternommen wird, eine neue kleine Zeitschrift historischen Charakters herauszugeben, so geschieht dies aus dem zuversichtlichen Glauben heraus, daß in schweren Zeiten die Besinnung auf vorhandenes Patrimonium einen lebendigen Faktor aufrechter Haltung darstellt und einen fruchtbringenden Quell innerer Kraft erschließen hilft. Das hat sich für die eigentlichen historischen Zeitschriften und Interessenkreise der Schweiz bereits vielfach erwiesen und ist einem erst kürzlich wieder bewußt geworden beim 100. Jubiläum der Allgemeinen Geschichtforschenden Gesellschaft der Schweiz. Aber gerade da hat sich auch gezeigt, wieweit die Gebiete der Medizin, der Naturwissenschaften und der Technik, soweit sie historische Substanz an sich haben, bei solchem schweizerischen Zusammenklang noch abseits stehen. Die relativ junge Existenz der modernen Naturwissenschaften, denen Medizin und Handwerk viel mehr zu Gevatter gestanden haben als die aus dem Altertum heraufgewachsene Philosophie, hat - neben Terminologie-Schwierigkeiten - es ganz natürlich mit sich gebracht, daß diese Gebiete von der eigentlichen Geschichtswissenschaft wenig gepflegt werden.

Solchem Mangel abzuhelfen und auch für die Schweiz über die Vortragstätigkeit unserer Gesellschaft hinaus ein kontinuierliches Kristallisationsund Vitalitätszentrum historischer Interessen innerhalb dieser Gebiete zu schaffen, ist die kleine Zeitschrift gedacht, die sich Conrad Gessner zum Patron wählt, ihn, den humanistischen Zürcher Arzt und Vater der Bibliographie, der in Basel promoviert und in Lausanne unter bernischer Oberhoheit zeitweise akademisch tätig gewesen ... Jedenfalls soll hier, wie bei den gewichtigeren Vorläufern, eine Auffassung des Historischen zur Geltung kommen, die sich nicht dadurch leiten lassen wird, daß etwas der Vergangenheit angehört, sondern danach sich richtet, ob und wieweit etwas, das auch mit Vergangenem zusammenhängt, lebendig und anregend gestaltet werden kann. Nur so ist fruchtbare Saat zu gewärtigen und nur insofern hat eine Neugründung in solcher Zeit und im Angesicht praktisch und nüchtern tätiger Wissenschaft und Technik ihre sinnvolle Berechtigung. Von solcher Warte aus kann dann vielleicht auch gehofft werden, daß die Spezialwissenschaften selbst, deren Geschichte hier in Frage steht, den sich stellenden Problemen gegenüber sich öffnen und zugänglich erweisen werden. Denn immer noch unerfüllt und verlockend klingt aus den Weltgeschicht- 
lichen Betrachtungen der fragend-werbende Ruf Jаков BuRcKhaRdTs herüber, ob dann die Naturwissenschaften, in denen er, zum Unterschied von der Philosophie, die einzigen uneigennützigen Kameraden der Historie sah, sich selbst von der Geschichte des Geistes ausschließen wollen. Darum sei auch in der Schweiz, von wo vor über einem halben Jahrhundert solcher Ruf zuerst erklang, ein Versuch in dieser Richtung gewagt.»

Wenn es dem Herausgeber der Neugründung einer Zeitschrift einigermaßen gelungen ist, die Zeitschrift bis ins 25. Jahr durchzuhalten und ihr einen nicht allzu engen Rahmen zu geben, so mußte er sich doch stets darüber Rechenschaft geben, wie eng die Grenzen seiner Tätigkeit gezogen waren und sind, welche ihm die finanziellen Möglichkeiten vorschreiben. Gerne hätte er den Inhalt der Zeitschrift erweitert und ihren Gehalt vertieft und wäre mit anderen, ähnlich gearteten Zeitschriften in näheren Kontakt getreten. Auch war es nur zeitweise möglich, bibliographische Übersichten über die in der Schweiz herauskommende medizin- und naturwissenschaftshistorische Publizistik periodisch zu referieren. Denn seit der Gründung der kleinen Zeitschrift hat sich der schweizerische Anteil am Aufbau der medizinischen und naturwissenschaftlichen Wissenschaftsgeschichte ganz wesentlich erweitert. An den meisten schweizerischen Universitäten sind Lehrstühle und Institute für Geschichte der Medizin und der biologischen Wissenschaften gegründet worden oder im Aufbau begriffen. Auch sind einige wenige Dozenturen für die Geschichte der Naturwissenschaften entstanden, und wir überblicken ein mehr oder weniger koordiniertes Zusammenspiel der Fachvertreter und - im weiteren Rahmen - der Förderer und Liebhaber dieser historischen Fachgebiete. Es besteht kein Zweifel, daß noch ein mehreres zu tun ist und daß noch viele ungehobene Schätze, die sich in öffentlichen Bibliotheken oder in privatem Besitz befinden, ihrer Aufschließung durch kompetente Gelehrte harren. Auch darin haben wir im Laufe der Jahre manchen schönen Aufschluß, etwa aus dem Nachlaß Conrad Geßners, Albrecht von Hallers oder Johann Jakob Wepfers, erleben dürfen. Aber wie vieles bleibt bei den eben Genannten noch zu tun, wieviel Unediertes enthalten die Nachlässe von Johann Jakob Scheuchzer, oder der Bauhin, Bonnet, Trembley, Tronchin, Tissot, Troxler, de Saussure, de Candolle, Louis Agassiz, Oswald Heer und vieler anderen.

Handelt es sich bei dieser Aufschließung im wesentlichen um die Verpflichtung, dem helvetischen Geistesleben früherer Zeiten auf den Gebieten der Medizin und der Naturwissenschaften im größeren Rahmen der allgemeinen Geschichte den Boden zu bereiten, so stellt uns die Wissenschafts- 
geschichte vor die nicht minder wichtige Aufgabe, die lebendige Beziehung zwischen Vergangenheit und Gegenwart herzustellen und auf diesem Wege den Sinn für das historische Verständnis zu wecken. Nur so schließt sich der Bogen von der Vergangenheit zur Gegenwart, die von der Zukunft bald eingeholt, selbst zur gelebten, erlebten Vergangenheit wird.

Dankbar anerkennt der Herausgeber des Gesnerus die Unterstützung, die er in den langen Jahren von schweizerischen und ausländischen Vertretern der medizinischen und naturwissenschaftlichen Wissenschaftsgeschichte stets erfahren durfte. Ganz besonders dankbar ist er den Mitgliedern der Schweizerischen Gesellschaft für Geschichte der Medizin und der Naturwissenschaften für ihr stets reges Interesse. Nur mit ihrem geistigen und materiallen Einsatz war es möglich, die Zeitschrift auch in kritischen Zeiten durchzuhalten. Der Dank gilt nicht minder der tatkräftigen Hilfe und moralischen Unterstützung, welche der Herausgeber des Gesnerus von privater Hand und durch öffentliche Institutionen empfangen durfte. Dies gilt in ganz besonderem Maße für die namhafte Unterstützung, welche die Vierteljahrsschrift Gesnerus durch die Schweizerische Naturforschende Gesellschaft, insbesondere in den letzten Jahren, erhalten hat.

Der Herausgeber des Gesnerus dankt allen, insbesondere den Mitgliedern der Schweizerischen Gesellschaft für Geschichte der Medizin und der Naturwissenschaften, für das Vertrauen, das sie ihm entgegengebracht haben, und er bittet sie darum, ihm auch in Zukunft dieses Vertrauen zu bewahren. Ein besonderer Dank gilt dem Verlag Sauerländer in Aarau, der in den 25 Jahren keine Mühe gescheut hat, die oft schwierigen Druckaufgaben in zuverlässigster Weise auszuführen. 